\title{
CÁTEDRA LIBRE DE PUEBLOS ORIGINARIOS. UNIVERSIDAD NACIONAL DE LA PATAGONIA SAN JUAN BOSCO: EXPERIENCIAS, INTERPELACIONES Y DESAFIOS
}

\author{
PUBLIC LECTURE ON NATIVE POPULATIONS. UNIVERSIDAD NACIONAL DE LA \\ PATAGONIA SAN JUAN BOSCO: EXPERIENCES, QUESTIONING AND CHALLENGES
}

Sonia Liliana Ivanoff ${ }^{1}$ - Daniel Leonidas Loncon $^{2}$

Fecha de recepción: 24-06-2016

Fecha de aceptación y versión final: 18-10-2016

Resumen: La Cátedra Libre de Pueblos Originarios fue creada en el año 2008, en el ámbito de la Secretaría de Extensión Universitaria, Universidad Nacional de la Patagonia "San Juan Bosco", y viene desarrollando proyectos de articulación, vinculación y promoción de los derechos de los pueblos originarios en la provincia del Chubut, Patagonia Argentina. Tiene como misión dar una respuesta social a demandas concretas realizadas a la Universidad por parte de las autoridades de las comunidades indígenas, conformando una propuesta curricular que ha ido adquiriendo una fisonomía particular, trascendiendo en un amplio espacio territorial.

En el siguiente artículo, nos proponemos dar cuenta de las experiencias, interpelaciones y desafíos que el equipo de cátedra enfrenta en lo cotidiano, pero que sobretodo les ha permitido la concreción de sus objetivos, entre ellos, constituirse en un espacio de educación intercultural en el ámbito universitario.

La diversidad cultural sustentada en valores y saberes tradicionales, como modos de aprendizajes, son los ejes centrales en las actividades y programas que desarrolla la Cátedra Libre de Pueblos Originarios (CLPO).

Sus distintas líneas de trabajo, tienen un denominador común, generar mayores espacios de visibilidad y de articulación de los pueblos originarios, espacios que se traducen en experiencias tanto hacia adentro de la "comunidad universitaria" (personal no docente, docente, estudiante, y funcionarios de gestión política) como hacia fuera de la Universidad, trasladando sus aulas a los territorios indígenas.

Palabras clave: jóvenes indígenas, pueblos indígenas; educación superior; derecho a la educación; interculturalidad.

Abstract: The Public Lecture on Native Populations was created in 2008, by the University Extension Secretariat of the Universidad Nacional de la Patagonia "San Juan Bosco", and has been developing several projects focused on articulating, linking and promoting the rights of native populations in the province of Chubut, Patagonia, Argentina.

\footnotetext{
${ }^{1}$ Profesora en Historia y Abogada. Es diplomada en Políticas Sociales, Perito en Antropología Aplicada y Mediación y resolución alternativa de conflictos. Se desempeña como docente universitaria y es responsable en la coordinación de la Cátedra Libre de Pueblos Originarios, dependiente de la Secretaría de Extensión de la Universidad Nacional de la Patagonia San Juan Bosco, donde tiene la responsabilidad principal del diseño, ejecución y evaluación de proyectos destinados a pueblos originarios, comunidades y sus miembros de Chubut y Santa Cruz, Patagonia. Argentina. Correo electrónico: soniaivanoff2@hotmail.com

${ }^{2}$ Miembro del Pueblo Mapuche. Auxiliar de la Cátedra Libre de Pueblos Originarios. Secretaría de Extensión Universitaria Universidad Nacional de la Patagonia "San Juan Bosco" (Argentina). Desarrolla actividades de gestión, planificación y ejecución de programas en el campo de lo social y comunitario. Correo electrónico: danielloncon@yahoo.com.ar
} 
The aim of this lecture is to provide a social answer to specific requests made by the authorities of indigenous communities to the University, and therefore proposing a curriculum that has adopted special features, spreading throughout a wide area.

This article is intended to describe all the experiences, questionings and challenges that are faced every day, but have specially allowed the achievement of all the objectives; such us becoming part of an area of intercultural education in the university.

Cultural diversity based on traditional knowledge and values and as a way of learning is the focus of the activities and syllabi developed by the Public Lecture on Native Populations.

The different lines of work have a common denominator, which is to promote greater spaces of visibility and articulation of the natives populations, spaces that translate into experiences both within the "university community" (non-teaching and teaching personnel, students and officials) and outside the University, moving the classrooms to indigenous lands.

Key words: Indigenous youth; indigenous populations; higher education; right to education; interculturality.

\section{Introducción}

La Cátedra Libre de Pueblos Originarios' - CLPO - fue creada en el año 2008, en la sede Comodoro Rivadavia de la Universidad Nacional de la Patagonia "San Juan Bosco", ubicada en la Provincia del Chubut, Patagonia Argentina.

Forma parte de un grupo de Cátedras Libres que dependen de la Secretaría de Extensión Universitaria (ámbito de Rectorado) y su surgimiento responde a una demanda social y de las organizaciones del pueblo mapuche tehuelche de la Provincia de Chubut.

Durante todo este tiempo, como propuesta curricular fue adquiriendo su fisonomía particular, la que trasciende el espacio territorial vinculante de la Sede de Comodoro Rivadavia (Provincia de Chubut). El equipo de la CLPO se propone dar cuenta de las experiencias, las interpelaciones y desafíos que se enfrenta en lo cotidiano, pero que sobretodo han permitido la concreción de sus objetivos, entre ellos, constituirse en un espacio de educación intercultural en el ámbito universitario.

La tarea no ha sido fácil, máxime si pensamos en la tradición universitaria en donde se inserta, que es negadora de la existencia de los pueblos indígenas en su matriz sociocultural y se concibe además como productora exclusiva de saberes válidos. Los desafíos se reproducen en experiencias propuestas desde la CLPO, surgidas de las interpelaciones recibidas, que buscan responder y romper con patrones de conocimiento estándar.

Las prácticas que se proponen tampoco responden a un esquema de Cátedra convencional universitaria, sino que más bien responden a criterios que promuevan proyectos de interrelación con miembros de pueblos indígenas, ponderando sus saberes.

La CLPO se ha propuesto articular distintas líneas de trabajo, que tienen como denominador común, generar mayores espacios de visibilidad y de articulación de los pueblos indígenas, tanto en experiencias hacia dentro de la comunidad universitariai como por fuera de la Universidad.

Las experiencias que se proponen giran en torno a tres perspectivas de análisis: la perspectiva histórica, que busca descolonizar y cuestionar prácticas que conducen a la desmemoria y la invisibilización de los pueblos indígenas en el relato histórico construido desde el poder del estado. Desde la perspectiva del derecho, el fuerte anclaje que han tenido en el orden jurídico el reconocimiento de los derechos humanos para los pueblos indígenas; derechos que brindan un encuadre favorable 
para que se den prácticas interculturales. Por último la perspectiva indígena, por una parte desde los pueblos indígenas en su vinculación con el Estado y, por otra, desde el reconocimiento de derechos hacia los pueblos indígenas.

En las provincias argentinas de Chubut y Santa Cruz, existen dos pueblos indígenas: Mapuche y Tehuelche, no obstante ello, la mayor parte de las comunidades se autoreconocen como Mapuche-Tehuelche en razón de identificar componentes ancestrales de ambos pueblos. Por tal motivo, en el presente escrito se utilizará la referencia Mapuche-Tehuelche para hacer alusión a los pueblos indígenas de la región patagónica.

Desde la CLPO, nos pusimos como objetivo prioritario posicionarnos sobre los hechos políticos más relevantes para los pueblos originarios y la sociedad toda, tales como: recuperación territorial, reconocimiento de derechos, tratamiento de los medios de comunicación y acceso a la justicia. Ello nos exige posicionarnos frente a los cambios jurídicos, sobre la inclusión de los nuevos sujetos de derecho y promover la necesidad de implementar las transformaciones que los hagan realidad.

\section{Antecedentes y contexto}

Desde la Constitución Nacional de 1853, las políticas de gobierno buscaron el exterminio y la desaparición de las comunidades indígenas de la región patagónica a través de campañas militares, como la mal denominada "Conquista del desierto". Una vez finalizada esta guerra, o conquista santa", se fueron consolidando políticas de sumisión, asimilación e integración. Los sobrevivientes fueron confinados a tierras marginales, mayormente improductivas, generalmente en forma de reducciones, reservas o colonias.

El enorme avance en el reconocimiento de los derechos humanos a nivel internacional y especialmente, el fin de la última dictadura militar, permitieron que el reclamo de pueblos originarios se reinstalase en la agenda pública. Este reclamo tomó particular impulso a partir de la reforma constitucional del año 1994, cuando el Estado argentino se proclamó jurídicamente como un Estado pluricultural que debía reparar históricamente el daño causado a los pueblos originarios e inscribió en la Constitución Nacional el reconocimiento de su preexistencia territorial, étnica y cultural. Al mismo tiempo, se da jerarquía constitucional (art. 75 inc. 22 de la $\mathrm{CN}$ ) a tratados internacionales de derechos humanos, otros instrumentos internacionales, como el Convenio 169 de la Organización Internacional del Trabajo (OIT), la Declaración de las Naciones Unidas sobre los Derechos de los Pueblos Indígenas, la Declaración de la ONU sobre los derechos de las personas Pertenecientes a Minorías Étnicas, religiosas y Lingüísticas, la Declaración de la Conferencia Mundial contra el Racismo, la Discriminación Racial, la Xenofobia y las Formas conexas de Intolerancia, y la Declaración Universal de la Organización de las Naciones Unidas para la Educación, la Ciencia y la Cultura (UNESCO) sobre Diversidad Cultural.

No obstante estos avances en materia normativa, no tienen el impacto esperado en la realidad de los reclamos territoriales de los pueblos indígenas de la región patagónica. Esta divergencia entre el "deber ser" y el "ser", determinó que sigan perpetrándose los abusos y las violaciones a los derechos humanos y territoriales. La 
asimetría existente, tiende a perpetuar la situación histórica de marginación, despojo y miseria de los pueblos indígenas.

En el Boletín Observatorio de Derechos Humanos de Pueblos Indígenas (ODHPI) iv surge como diagnóstico que la imposibilidad por parte de las comunidades indígenas y sus miembros de acceder a la justicia para la defensa o reclamo de sus derechos territoriales está ligado a la dificultad real de sus miembros de enfrentar los obstáculos que presentan los complejos trámites administrativos y judiciales. La lentitud y burocracia de los procedimientos administrativos y judiciales torna difícil garantizar el acceso a la justicia que se proclama como garantía a los pueblos indígenas.

Este cuadro de situación que atraviesan los pueblos indígenas son algunos de los aspectos que se tienen en cuenta y que permitirán con el tiempo modificar prácticas colonizantes que se observan en el sistema educativo. Si bien las Universidades hacen sus esfuerzos para resolver algunos de estos problemas, resulta menester no desconocer la cotidianidad, la diversidad cultural. Por ello se realizan alianzas estratégicas, no sólo con las organizaciones indígenas sino además con otras instituciones universitarias $\mathrm{y} / \mathrm{o}$ instituciones gubernamentales y no gubernamentales.

Al analizar las diversas dificultades que los miembros de comunidades indígenas manifiestan, el territorio aparece como el eje de trabajo preponderante. La falta de acceso a la justicia de las comunidades indígenas y sus miembros, en lo referente al conocimiento y defensa de sus derechos territoriales, contribuye a configurar un marco de permanentes violaciones de tales derechos y al hacerlo, se vulneran otros como sus derechos a la alimentación, a la vivienda digna, al desarrollo cultural, a la salud, a educación y a la identidad. La sociedad ignora otras visiones del mundo, lenguas, conocimientos y proyectos que para su futuro proponen los pueblos originarios. Proyectos en los que expresan la diversidad cultural que los caracteriza.

Además del marco de reconocimiento de derechos vigente es propicio señalar y necesario que como institución universitaria debemos necesariamente enmarcar nuestras actividades en concordancia con los acápites C3 y D4 de la Declaración Final de la Conferencia Regional de Educación Superior en América Latina y el Caribe (Cartagena, 2008) que establecen "promover la diversidad intercultural" y "fortalecer lo pluricultural".

La CLPO procura, a través de sus actividades, promover la diversidad cultural y la interculturalidad en la Educación Superior, con el propósito de reafirmar y fortalecer el carácter pluricultural, multiétnico y multilingüe del espacio territorial en que se encuentra inserta la Universidad Nacional de la Patagonia San Juan Bosco (UNPSJB).

\section{Acciones y propuestas llevadas a cabo desde la Cátedra Libre de Pueblos Originarios}

En este contexto, la CLPO se propuso entre sus principales acciones:

a) Convenios específicos para el fortalecimiento de redes institucionales: Se suscribieron convenios con los Poderes Ejecutivo y Judicial: Instituto Nacional contra la Discriminación, la Xenofobia y el Racismo (INADI) y Ministerio de la Defensa Pública de Chubut, Hospital Rural de Cushamen, Organizaciones de defensa de derechos humanos como la Asociación Argentina Abogados y Abogadas de Derecho Indígena 
(AADI) y se estrecharon vínculos con universidades de América Latina mediante la Red de Educación Superior y Pueblos Indígenas (Red ESIAL).

b) Publicaciones de material referido a temáticas indígenas por medio de la editorial de la Universidad.

c) Cursos y Seminarios de capacitación en diversas Facultades de la UNPSJB e Instituciones de Formación Docente de la Provincia de Chubut.

d) Proyectos de investigación y divulgación sobre la temática.

Asimismo, se desarrollan estrategias específicas, tendiente al logro de los objetivos:

- Curso de Promotores Jurídicos Indígenas, desde donde se propone capacitar a través del protagonismo y la participación activa a los integrantes de las comunidades indígenas, estudiantes y público en general interesados en la temática.

El curso pretende ser una herramienta que fortalezca a las comunidades originarias en la defensa y la reivindicación de sus derechos territoriales. El Curso ha tenido un impacto significativo entre los participantes, generando una constante demanda para que sea replicado en otros contextos comunitarios y en particular hacia dentro de la propia Universidad.

Además, cabe mencionar que las diversas propuestas surgen del análisis de las principales demandas de los propios actores, tendientes a la promoción comunitaria y al desarrollo intercultural dentro de la educación superior.

- Revitalización y revalorización de Idiomas originarios

Del cuadro de situación descripto, existe una demanda constante de la enseñanza de los idiomas originarios, como parte constitutiva de la identidad de los pueblos indígenas y la diversidad cultural en la que se inserta la Universidad Nacional de la Patagonia "San Juan Bosco" (en adelante UNPSJB). Es por ello que se desarrollan dos cursos:

- Idioma Mapuzungun (Idioma del Pueblo Mapuche): Se dicta desde el año 2009.

- Idioma Guaraní: En la actualidad se desarrollan dos actividades de capacitación, una de enseñanza propiamente dicha de Idioma Guaraní en las aulas de la Universidad, y otra en el "Centro de Apoyo Escolar Bilingüe", ubicado en un barrio de la ciudad. Ello responde a la fuerte presencia de hablantes de idioma guaraní en la ciudad de Comodoro Rivadavia.

A su vez, ambas propuestas tienen su correlato con sendos programas en la radio de la Universidad.

- Programas de Radio

Las diversas propuestas de la CLPO se difunden por medio de dos programas de radio en la emisora de frecuencia modulada de la Universidad (FM 93.1 Universidad).

- Proyecto de Aprovisionamiento de agua a Comunidades Indígenas ${ }^{v}$

A través de la vinculación con el Programa de Responsabilidad Social Empresaria, y a fin de dar respuesta a un requerimiento social realizado por las Comunidades Indígenas, para el aprovisionamiento de agua, desde la CLPO se realizó el diseño, gestión y ejecución de un Proyecto que consiste en la instalación de tanques y molinos para la acumulación de agua. Este trabajo es llevado adelante por las propias comunidades a través del trabajo autogestivo.

- Difusión de los derechos 
Se han dictado charlas, conferencias y seminarios hacia el interior de la Universidad y de Institutos de Formación Docente de la ciudad de Comodoro Rivadavia (Profesorados para nivel inicial y primario) a los fines de acompañar los procesos de visibilización de los derechos indígenas con un cambio de paradigma de reconocimientos de los derechos constitucionales para los pueblos indígenas ${ }^{\mathrm{vi}}$.

- Seminarios sobre actualidad de los pueblos indígenas en la Provincia del Chubut.

Mediante acuerdos con diversas Facultades de la Universidad, se dictan seminarios bajo la modalidad de talleres participativos con formación intercultural en un marco de intercambio de experiencias con miembros de comunidades y/o pueblos indígenas.

De igual modo, se han realizado sugerencias de contenidos específicos sobre la temática para que sean incorporados a los contenidos curriculares de las diversas carreras.

- Publicaciones

Como producto del proceso de visibilización del tema, se han producido dos publicaciones de divulgación; una sobre Mapuzungun (idioma del Pueblo Mapuche), que pretende brindar herramientas básicas para iniciarse en el dominio de la lengua e interactuar en situaciones comunicativas sencillas; y un Dossier de Propiedad Comunitaria Indígena, que condensa algunas de las principales intervenciones de miembros de la Asociación de Abogados de Derecho Indígena, en una materia que desde la reforma de la constitución nacional en 1994, estableció el reconocimiento a del derecho de propiedad comunitaria, para las comunidades de pueblos indígenas.

- Proyectos de Investigación

Actualmente se encuentran en curso dos proyectos de investigación:

a) "Situación sociolingüística y sociocultural de los pueblos originarios ubicados en la parte del corredor Bioceánico. De Gulu Mapu a Puel Mapu".

b) Historia del Hospital Rural de Cushamen. Proyecto llevado a cabo por la Cátedra antes referida y diversos profesionales de la región. Pretende indagar acerca del surgimiento de la primera institución estatal en el territorio indígena denominado posteriormente como Cushamen.

Asimismo, se desarrolló, en conjunto con el Instituto Nacional contra la Discriminación, la Xenofobia y el Racismo (INADI) el Mapa de la Discriminación para la provincia de Chubut, un estudio sobre las percepciones, representaciones y experiencias en torno a la discriminación.

\section{Interpelaciones y desafíos}

La educación superior en nuestro país y en nuestra región patagónica en particular, se encuentra frente a desafíos contemporáneos muy particulares. En dos años se cumplirá un siglo de la Reforma Universitaria de 1918, la que consagró los principios de autonomía, cogobierno y la extensión universitaria como principales conquistas del movimiento estudiantil de aquella época.

En el caso de la Cátedra Libre de Pueblos Originarios, la misma es creada en el ámbito de la Secretaría de Extensión Universitaria, lo cual permite una vinculación 
directa con organizaciones indígenas, estudiantes y docentes universitarios pertenecientes a pueblos originarios.

Desde el año 2008 hasta la actualidad, la Cátedra se ha consolidado como un espacio de participación, debate y consulta en virtud del trabajo constante y sostenido en el tiempo; ha sido dúctil en función de las demandas del medio, ya sean de tipo académicas o las planteadas por las comunidades y organizaciones indígenas con las cuales se trabaja.

Al promover actividades que estimulan a que se incorporen al currículum, los "saberes interculturales", la CLPO contribuye a mejorar la calidad de la Universidad y otras instituciones de educación superior en donde debe aparecer la visión de lo diverso, visiones del mundo y conocimientos, lenguas, historias, problemas y propuestas de los pueblos originarios.

Es menester señalar el respaldo institucional de las autoridades de turno que han favorecido procesos de participación indígena al interior de la Universidad, mediante el desarrollo de diversos cursos, seminarios, propuestas de extensión de cátedra, participación en eventos como aniversarios de la Universidad, exposiciones, entre otras; como también el acompañamiento de las actividades de la Cátedra Libre en territorio indígena.

Actualmente nos encontramos en un proceso de sistematización de información sobre los niveles de autoreconocimiento de pertenencia a pueblos originarios y afrodescendientes de los miembros de la comunidad universitaria, con el objetivo de proponerles diversas actividades en donde puedan ser actores protagónicos frente a los nuevos diálogos de la Educación Superior con los pueblos originarios.

De igual forma, la constitución de grupos de estudio e investigación con estudiantes y docentes indígenas, aparece como un horizonte necesario a los efectos de recuperar los conocimientos perdidos o distorsionados por el poder hegemónico, esto implica, como lo señala Claudia Zapata, una "reescritura de los sujetos colonizados", permitiendo sumergirse en el pasado, cuyo ejercicio permitirá resignificar el ser y estar en el mundo. En este caso particular, con una historia reciente de conquista militar cuyos efectos devastadores aún se encuentran muy latentes.

La reflexión, como jóvenes indígenas contemporáneos en un contexto universitario, implica tener en cuenta múltiples variables, entre las que se encuentran:

a) el proceso del auto reconocimiento. Reconocerse como parte de un pueblo originario conlleva un proceso de revisión interna de las propias historias y trayectorias. Cuando se indaga en las historias y tradiciones familiares, aparecen retazos y trozos de una carta que se escribió, se escribe y aún tiene espacio en blanco. Es encontrarse con respuestas, para las cuáles muchas preguntas aún no se han formulado, por lo que no deja de ser un hito fundamental en la historia del sujeto y su entorno inmediato.

b) El encuentro con el Otro. A partir de asumir la identidad, se configura una nueva forma de relación con los otros. El periodista Pedro Cayuqueovii, comenta que no se dio cuenta de su otredad cultural hasta tanto no salió de su comunidad y se encontró con otros, que le dijeron que era diferente. Esto nos permite pensar en las trayectorias personales y particulares de los jóvenes indígenas que llegan a la Universidad, en la mayoría de los casos, provenientes de comunidades pequeñas y contextos socioculturales distintos. El encuentro con el otro, de algún modo, es 
también un encuentro con sí mismo, dado a que ese encuentro implica compartir y poner de relieve historias propias y un diálogo profundo sobre la trayectoria y una subjetividad en constante transformación.

c) La resignificación de las prácticas y el pensamiento. A partir del auto reconocimiento y el encuentro con el otro se resignifican considerablemente los conocimientos aprehendidos hasta el momento. Las prácticas, sentidos y lo discursivo se enfrentan a una nueva realidad revelada, a veces de forma abrupta e inesperada. Emergen nuevas formas de entender el mundo que nos rodea y las interpretaciones de los hechos e historias, hasta el momento incólumes, son sometidas a nuevas visiones e interpretaciones. Comprender y sentirse parte de una historia, que sufrió múltiples distorsiones y manipulaciones en desmedro de ese colectivo al que se adscribe, genera múltiples sensaciones para las cuáles aún no se tiene la reacción o respuesta adecuada. La primera sensación de injusticia y despojo histórico aparecen como las puntas de un iceberg que irá revelando nuevas verdades.

d) La revalorización de lo propio: Luego de las primeras impresiones y sensaciones, emerge un profundo sentido reivindicatorio de los valores, saberes y experiencias en torno a las vivencias del Pueblo. Reivindicación que puede tornarse en actos de militancia activas, de muy variadas formas, o simplemente una valorización más introspectiva.

Por lo que el autoreconocimiento de los jóvenes en un contexto universitario es de fundamental importancia dado al momento particular de sus trayectorias vitales.

Como equipo, consideramos que estas acciones son necesarias para remediar no sólo las inequidades que afectan especialmente a dichos pueblos, sino también algunos problemas que afectan la calidad de vida y la sostenibilidad social y ambiental de las respectivas sociedades en su conjunto.

Actualmente el Pueblo Mapuche-Tehuelche está ejerciendo acciones de reivindicación territorial, las cuales han sido blanco de ataques y criminalización mediática desde diversos sectores.

Ante lo cual, se hace aún más imprescindible que desde la Universidad se generen debates de ideas y se visibilice a la población indígena que acude a la misma, a los efectos de no reproducir prácticas discriminatorias o que atenten contra el proceso de auto identificación. En consonancia con el análisis que trabaja hace muchos años Daniel Matoviii , claramente nos posicionamos en que ningún saber es universal, y necesariamente es imprescindible la "colaboración intercultural".

Por ello al ver las carencias en la desvalorización del conocimiento indígena en nuestra región, y en particular en el sistema educativo, donde las múltiples experiencias de vida de los pueblos indígenas han resultado interculturales - de hecho y a la fuerza- ix; experiencias a partir de las cuales se pueda mostrar a la sociedad en general, valores y saberes interculturales. Estos pueblos se han apropiado y reelaborado la idea de interculturalidad para formular interpretaciones de sus propias experiencias de vida en el interior de las sociedades, buscando no ser convertidos en meras traducciones de lo que interpretan "otros" sobre ellos.

Conscientes de que existen problemas en reconocer el derecho a una "educación propia" a los pueblos indígenas, pareciera que fuera un objetivo inalcanzable. Lo que debemos asociarlo, en algunos casos, a la fuerte desvalorización de los saberes 
propios -no académicos- $y$, en otros casos, se admiran bajo el riesgo de ser folklorizados o son subordinados.

Si bien hay una escalada en el reconocimiento del derecho de los pueblos indígenas, en materia de Educación Superior, podríamos asegurar, por "colaboración intercultural" que se ha creado junto con miembros de las comunidades indígenas de Chubut y Santa Cruz, que sin ella es imposible comprender los significados históricos, jurídicos, políticos, económicos, sociales y otros. La falta de este tipo de colaboración hace que nuestra sociedad sea vista de manera fragmentada.

La promoción de la diversidad cultural, la incorporación de "saberes indígenas", intelectuales indígenas en el campo de la cultural y política son importantes, se convierten en los desafíos y retos, que para el equipo de la CLPO son el horizonte de nuestro trabajo.

Esto guarda relación con lo dispuesto en el Convenio 169 de la OIT, sobre las garantías del derecho a la participación y el carácter obligatorio de la consulta libre, previa e informada para los pueblos indígenas en los asuntos que les conciernen.

A pesar de las situaciones adversas, estamos convencidos que es un logro seguir incorporando a modo de diálogo, los saberes indígenas y los saberes científicos, tanto para la comunidad universitaria como para los miembros de las comunidades y pueblos indígenas. El reconocer la diversidad de valores y los distintos modos de aprendizajes son elementos centrales en la elección de las actividades y programas que desarrolla la CLPO, y que se seguirán propiciando.

Otro desafío es poder continuar con el trabajo territorial, para que la Universidad, también pueda comprender nuevas lógicas, formas de ver el mundo y de concebir el conocimiento.

Otros retos, que nos hemos propuesto, tienen que ver con interculturalizar los diseños curriculares y que los programas de estudio de las diversas carreras universitarias incorporen contenidos vinculados a los pueblos originarios, no en función de su especificidad profesional, sino teniendo en cuenta el heterogéneo universo de alumnos que una Universidad alberga y particularmente por la región geográfica en la que nos encontramos.

\section{Consideraciones finales}

El contribuir a cuestionar las inequidades que afectan en especial a los pueblos indígenas (dentro y fuera de la comunidad universitaria), se ha convertido en un objetivo posible de cumplir. No obstante, para ello, somos conscientes que hay mucho por hacer. Aún resta instalar en el ámbito académico otros conocimientos, que sean incorporados a los currículos: cosmovisiones, lenguas, historias, conocimientos tradicionales y los problemas y propuestas que los pueblos indígenas plantean.

Los desafíos deben orientarse a mejorar las posibilidades de acceso, a la apropiada formación y graduación de estudiantes indígenas, que repercutirá sin lugar a dudas en el mejoramiento de la calidad de vida y en la sostenibilidad social y ambiental de la sociedad de la que forman parte.

\section{Bibliografía}


Bayer, O. (2010) Historia de la crueldad argentina: Julio A. Roca y el genocidio de los pueblos originarios. Buenos Aires, El Tugurio.

Briones, C. (2008) Diversidad cultural e interculturalidad: ¿de qué estamos hablando? en García Vázquez, C. Hegemonía e interculturalidad. Poblaciones originarias y migrantes. La interculturalidad como uno de los desafíos del siglo XXI. Prometeo Libros, Buenos Aires.

Mato, D. (2008) No hay saber "universal", la colaboración intercultural es imprescindible", En Revista Alteridades, vol.18, núm.35, 101-116.

Montenegro, M. (2015) Colaboración intercultural y Arqueología: nuevas propuestas para la Educación Superior en el nordeste de Argentina, en: Mato, D. Educación Superior y Pueblos Indígenas en América Latina: Contextos y experiencias. Universidad Nacional de Tres de Febrero, Sáenz Peña, Buenos Aires.

Zapata, C. (2013) "Lo particular y lo universal en el pensamiento de Franz Fanon. Contrapunto con la intelectualidad indígena contemporánea" en: Stecher, L.; Zapata, C. y E. Oliva Franz Fanon desde América Latina. Lecturas contemporáneas de un pensador del Siglo XX. Buenos Aires, Corregidor.

\section{Notas:}

${ }^{i}$ En Argentina se utilizan tanto los términos Pueblos Indígenas como Pueblos Originarios en referencia a los pueblos preexistentes a los Estado Nación.

"Entendemos por "comunidad universitaria" al personal docente, no docente, estudiantes y funcionarios de gestión política.

iii Un artículo del diario la Prensa del 16/10/1878 decía: "la conquista es santa; porque el conquistador es el bien y el conquistado, el Mal".

iv Observatorio de Derechos Humanos de Pueblos Indígenas, Boletín comunicacional, Neuquén, 2013.

v Video del Proyecto: http://www.infoweb3.unp.edu.ar/extension/

vi Video Derechos de los Pueblos Originarios Parte 1: www.youtube.com/watch?v=jG06xipTgEw

vii Cayuqueo Pedro, Solo por ser indios y otras crónicas mapuches.

viii Daniel Mato, "No hay saber 'universal', la colaboración intercultural es imprescindible", en Alteridades 18 (35) 2008, pp. 101-116 y Mónica Montenegro, "Colaboración Intercultural y Arqueología Pública: nuevas propuestas para la educación Superior en el noroeste de Argentina", En: Mato, D. (coord.) "Educación Superior y Pueblos Indígenas en América Latina. Contextos y experiencias." (2015). Eduntref. Bs.As. pp. 207-218

${ }^{\text {ix }}$ Daniel Mato; Op. Cit. pp.103 